\title{
ARTICLE OPEN Predicting Dirac semimetals based on sodium ternary compounds
}

\author{
Bo Peng ${ }^{1,2}$, Changming Yue ${ }^{1,3}$, Hao Zhang ${ }^{2}$, Zhong Fang ${ }^{1,4}$ and Hongming Weng $\mathbb{D}^{1,4,5}$
}

Predicting new Dirac semimetals, as well as other topological materials, is challenging since the relationship between crystal structure, atoms and band topology is complex and elusive. Here, we demonstrate an approach to design Dirac semimetals via

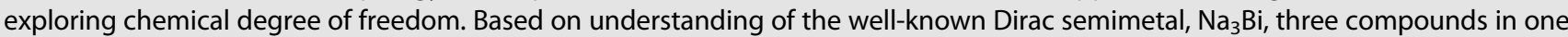
family, namely $\mathrm{Na}_{2} \mathrm{MgSn}, \mathrm{Na}_{2} \mathrm{MgPb}$, and $\mathrm{Na}_{2} \mathrm{CdSn}$, are located. Furthermore, hybrid-functional calculations with improved accuracy for estimation of band inversion show that $\mathrm{Na}_{2} \mathrm{MgPb}$ and $\mathrm{Na}_{2} \mathrm{CdSn}$ have the band topology of Dirac semimetals. The nontrivial surface states with Fermi arcs on the (100) and (010) surfaces are shown to connect the projection of bulk Dirac nodes. Most importantly, the candidate compounds are dynamically stable and have been experimentally synthesized. The ideas in this work could stimulate further predictions of topological materials based on understanding of existing ones.

npj Computational Materials (2018)4:68; doi:10.1038/s41524-018-0124-5

\section{INTRODUCTION}

Dirac semimetals (DSMs) ${ }^{1-5}$ are the three-dimensional (3D) analogs of graphene ${ }^{6}$ with and only with Dirac nodes on the Fermi level. These Dirac nodes are formed by band crossing, and the low-energy excitation around them leads to quasiparticles described by Dirac equation as emergent massless Dirac fermions. ${ }^{5,7-11}$ Up to now, there have been three classes of DSM proposed. One is the Dirac nodes with fourfold essential degeneracy, which is enforced by the nonsymmorphic symmetry at the high-symmetric momenta on the boundary of the Brillouin zone. ${ }^{1}$ The second is the accidental degenerate Dirac nodes, which appears as the topological phase transition critical point between different topological insulating states. ${ }^{12}$ The third one is also an accidental DSM, but the band crossing points are caused by band inversion and protected by proper crystal symmetry. ${ }^{2,11}$ DSMs serve as a singular point of various topological states, such as topological insulators, Weyl semimetals, nodal line semimetals, and triple-point semimetals. ${ }^{13}$ DSMs exhibit many novel properties, such as high carrier mobility, ${ }^{14}$ unique surface states with Fermi arcs, ${ }^{2,15}$ and negative longitudinal magnetoresistivity due to the chiral anomaly. ${ }^{16,17}$

The breakthrough in the search for stable DSMs ${ }^{11}$ is achieved in the series of studies on $\mathrm{Na}_{3} \mathrm{Bi}^{2,4}$ and $\mathrm{Cd}_{3} \mathrm{As}_{2}$, ${ }^{3,18-21}$ both of which were first proposed through first-principles calculations. They present good examples of the realization of the DSM in the above third class. The Dirac nodes are induced by band inversion and protected by proper axial rotational symmetry. ${ }^{2,11}$ Such protection makes the Dirac nodes quite robust within a finite range of Hamiltonian parameters, which is exactly the reason why this class of DSM is experimentally available while the other two remain to be found.
Despite the success in identifying $\mathrm{Na}_{3} \mathrm{Bi}$ and $\mathrm{Cd}_{3} \mathrm{As}_{2}$ and the intensive studies on them, to identify more DSMs remains a big challenge. How to locate a specific material among thousands of known compounds is not clear. Here, we demonstrate a chemically intuitive approach for searching new DSMs to show the underlying physics and ideas. We choose the first DSM $\mathrm{Na}_{3} \mathrm{Bi}$ as a model system for tuning the chemical degree of freedom. Three sodium ternary compounds, $\mathrm{Na}_{2} \mathrm{MgSn}, \mathrm{Na}_{2} \mathrm{MgPb}$, and $\mathrm{Na}_{2} \mathrm{CdSn}$, are naturally selected. Further theoretical calculations reveal that the chemical trend in the elements of the same column in periodic table plays an important role in band inversion. The proposed general design principle can be used for finding new DSMs, as well as other topological materials.

\section{RESULTS AND DISCUSSION}

Material design

The crystal structure of $\mathrm{Na}_{3} \mathrm{Bi}^{2,7}$ can be viewed as the $A B$ stacking of honeycomb layers along the $c$-axis, as shown in Fig. 1(a). For each honeycomb layer, one $\mathrm{Na}(1)$ atom and one $\mathrm{Bi}$ atom take the $A$ and $B$ sub-lattice site, respectively. There are two additional $\mathrm{Na}$ (2) atoms above and below the $\mathrm{Na}(1)-\mathrm{Bi}$ honeycomb layer to connect the $\mathrm{Bi}$ atoms in the neighboring layers. As a wellunderstood DSM, its low-energy electronic band structure has been found to be mostly determined by the $\mathrm{Na}(1)$ and $\mathrm{Bi}$ atoms in the honeycomb layer. The two crossing bands along the $\Gamma-A$ direction forming Dirac nodes are dominated by $\mathrm{Na}(1)-s$ orbitals and $\mathrm{Bi} 6 p_{x, y}$ orbitals. ${ }^{2}$ At $\Gamma$ point the $\mathrm{Na}(1)-s$ bands are lower than those of $\mathrm{Bi} 6 p_{x, y}$ mainly due to two things. One is that the heavy $\mathrm{Bi}$ has a relatively high on-site energy for $6 p$ orbitals. The other is the interlayer coupling leads to splittings between the bonding and

\footnotetext{
${ }^{1}$ Beijing National Laboratory for Condensed Matter Physics, and Institute of Physics, Chinese Academy of Sciences, Beijing 100190, China; ${ }^{2}$ Department of Optical Science and Engineering and Key Laboratory of Micro and Nano Photonic Structures (Ministry of Education), Fudan University, Shanghai 200433 , China; ${ }^{3}$ University of Chinese Academy of Sciences, Beijing 100049, China; ${ }^{4}$ Collaborative Innovation Center of Quantum Matter, Beijing, China and ${ }^{5}$ Songshan Lake Materials Laboratory, Dongguan, Guangdong 523808, China

Correspondence: Hao Zhang (zhangh@fudan.edu.cn) or Hongming Weng (hmweng@iphy.ac.cn)

These authors contributed equally: Bo Peng, Changming Yue
}

Received: 4 July 2018 Accepted: 6 November 2018

Published online: 04 December 2018 

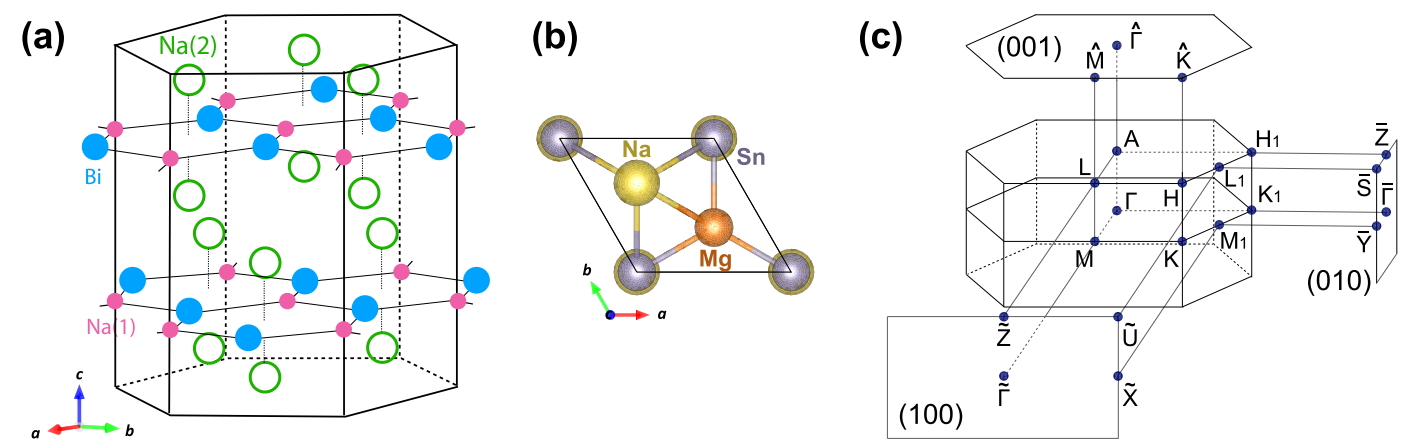

Fig. 1 a Crystal structure of $\mathrm{Na}_{3} \mathrm{Bi}$ with $\mathrm{Na}(1), \mathrm{Na}(2)$, and $\mathrm{Bi}$ sites indicated. b Top view of the $\mathrm{Na}_{2} \mathrm{MgSn}$ unit-cell with $\mathrm{Mg}$ and $\mathrm{Sn}$ replacing $\mathrm{Na}$ (1) and $\mathrm{Bi}$ atoms in a, respectively. c The bulk Brillouin zone and the projected surface Brillouin zone for (100), (010), and (001) surfaces

Table 1. Optimized lattice constants, and lengths of the two shortest bonds (in-plane $\mathrm{Mg} / \mathrm{Cd}-\mathrm{Sn} / \mathrm{Pb}$ bonds and vertical $\mathrm{Na}-\mathrm{Sn} / \mathrm{Pb}$ bonds) for $\mathrm{Na}_{2} \mathrm{MgSn}, \mathrm{Na}_{2} \mathrm{MgPb}$, and $\mathrm{Na}_{2} \mathrm{CdSn}$

\begin{tabular}{lllll}
\hline & $a(\AA)$ & $c(\AA)$ & $d_{\text {IIIV }}(\AA)$ & $d_{\text {Na-IV }}(\AA)$ \\
\hline $\mathrm{Na}_{2} \mathrm{MgSn}$ & 5.078 & 10.112 & 2.932 & 3.336 \\
& $\left(5.049^{29}\right)$ & $\left(10.095^{29}\right)$ & $\left(2.915^{29}\right)$ & $\left(3.328^{29}\right)$ \\
$\mathrm{Na}_{2} \mathrm{MgPb}$ & 5.157 & 10.240 & 2.977 & 3.375 \\
& $\left(5.110^{30}\right)$ & $\left(10.171^{30}\right)$ & $\left(2.950^{30}\right)$ & $\left(3.377^{30}\right)$ \\
$\mathrm{Na}_{2} \mathrm{CdSn}$ & 5.068 & 10.152 & 2.926 & 3.366 \\
& $\left(4.990^{31}\right)$ & $\left(10.111^{31}\right)$ & & \\
\hline The experimental data are presented in parentheses for comparison
\end{tabular}

anti-bonding states for both $s$ and $p$ bands along $\Gamma-A$. These two crossing bands with different orbital characters have different irreducible representations along the $\Gamma$-A direction and the Dirac nodes are protected.

Inspired by the above understanding, we notice that $\mathrm{Na}_{3} \mathrm{Bi}$ can be regarded as $\mathrm{Na}_{2} \mathrm{Na}_{1} \mathrm{Bi}$. The first two $\mathrm{Na}$ are on $\mathrm{Na}(2)$ site, which support the 3D lattice structure and also supply two electrons to the $\mathrm{Na}(1)-\mathrm{Bi}$ honeycomb layer. If the crystal structure and the electronic structure are similar to those of $\mathrm{Na3} B \mathrm{Bi}$, one can get a new DSM material. Thus, this leads to the idea to find other potential DSMs by simply changing the atoms in the $\mathrm{Na}(1)$-Bi layer. To induce band inversion, $\mathrm{Bi}$ should be substituted with other similar heavy metal atoms such as $\mathrm{Pb}$ and $\mathrm{Sn}$. Since $\mathrm{Pb}$ and $\mathrm{Sn}$ have one fewer valence electron than $\mathrm{Bi}$, to maintain the same band-filling, $\mathrm{Na}(1)$ should be substituted with atoms having twovalence electrons, such as alkaline-earth metal and II-B elements like $\mathrm{Mg}, \mathrm{Ca}, \mathrm{Sr}, \mathrm{Zn}, \mathrm{Cd}$, and $\mathrm{Hg}$. Thus, three sodium-containing ternary compounds reported experimentally, namely $\mathrm{Na}_{2} \mathrm{MgSn}$, $\mathrm{Na}_{2} \mathrm{MgPb}$, and $\mathrm{Na}_{2} \mathrm{CdSn}$, are naturally and immediately located. $\mathrm{Na}_{2} \mathrm{MgSn}$ and $\mathrm{Na}_{2} \mathrm{MgPb}$ have been successfully synthesized recently, ${ }^{22,23}$ while $\mathrm{Na}_{2} \mathrm{CdSn}$ has been synthesized and investigated in $1980 .^{24}$

Similar to $\mathrm{Na}_{3} \mathrm{Bi}$, all these compounds crystallize in hexagonal lattice with the space group $P 6_{3} / m m c\left(\# 194, D_{6 h}^{4}\right)$. We take $\mathrm{Na}_{2} \mathrm{MgSn}$ as an example, as demonstrated in Fig. 1(b). There are four $\mathrm{Na}$ atoms, two $\mathrm{Mg}$ atoms, and two $\mathrm{Sn}$ atoms in each unit-cell. The shortest bonds are those in the Mg-Sn layer. $\mathrm{Na}$ and $\mathrm{Sn}$ atoms align along the $c$-axis connected by the second shortest bonds. The optimized lattice constants and bond lengths are listed in Table 1, which are in good agreements with previous experimental results. ${ }^{22-24}$

For future experimental explorations, the stability of these three structures is an important aspect. ${ }^{25-27} \mathrm{~A}$ material is dynamically stable when there is no imaginary phonon frequency existing in its phonon spectrum. As shown in Fig. 2, no imaginary phonon frequency is found in all three materials, indicating their dynamical stability at $0 \mathrm{~K}$. This is consistent with the existence of them reported by experiments. As possible candidates for DSMs, one main advantage of these sodium ternary compounds compared to $\mathrm{Na}_{3} \mathrm{Bi}$ is structural dynamic stability. For $\mathrm{Na}_{3} \mathrm{Bi}$, the $P 6_{3} / m m c$ phase has been found dynamically unstable at the ground state due to large imaginary phonon frequencies. ${ }^{28}$ In fact, even now the ground state of $\mathrm{Na}_{3} \mathrm{Bi}$ is still under debate. ${ }^{29,30}$

\section{Electronic structures}

The calculated electronic structures of all three materials using the Perdew-Burke-Ernzerhof (PBE) functional and the Heyd-ScuseriaErnzerhof (HSE) hybrid-functional are shown in the top and middle panels of Fig. 3, respectively. The fatted bands with the weight of projected atomic orbitals are also shown in the middle panel for each of them. We focus on the band structures along $\Gamma-A$, where the band inversion and Dirac nodes happen in $\mathrm{Na}_{3} \mathrm{Bi}$.

In general, the strength of band inversion between the bands composed of $s$ orbitals (of $\mathrm{Mg}$ or $\mathrm{Cd}$ on $\mathrm{Na}(1)$ site) and $p$ orbitals (of $\mathrm{Sn}$ or $\mathrm{Pb}$ on $\mathrm{Bi}$ site) follows the order of total atomic number (mass) of the atoms in the unit-cell within both PBE and HSE calculations. The overestimation of band inversion in PBE is improved by HSE calculation. One can find that the lightest $\mathrm{Na}_{2} \mathrm{MgSn}$ has no band inversion and it is a normal semiconductor in HSE case. $\mathrm{Na}_{2} \mathrm{MgPb}$ has the same total mass as $\mathrm{Na}_{3} \mathrm{Bi}$ and is slightly lighter than the heaviest $\mathrm{Na}_{2} \mathrm{CdSn}$, but all of them have the similar band inversion along $\Gamma-\mathrm{A}$.

The spin-orbit coupling (SOC) is further included and the band structures of them are shown in the bottom panel in Fig. 3. Both $\mathrm{Na}_{2} \mathrm{MgPb}$ and $\mathrm{Na}_{2} \mathrm{CdSn}$ are DSMs with Dirac nodes on the path $\Gamma-$ $\mathrm{A}$, while $\mathrm{Na}_{2} \mathrm{MgSn}$ is an indirect band gap of $0.13 \mathrm{eV}$. For $\mathrm{Na}_{2} \mathrm{MgPb}$ and $\mathrm{Na}_{2} \mathrm{CdSn}$, one notable difference from $\mathrm{Na}_{3} \mathrm{Bi}$ is that there are two pairs of Dirac nodes since the one s-orbital band inverts with both the bonding and anti-bonding $p_{x, y}$-orbital bands. The $s$-band belongs to $\Gamma_{7}$ representation while the two $p_{x, y}$ bands belong to $\Gamma_{9}$ representation. The splitting in the bonding and anti-bonding $p_{x, y}$ (in-plane orbitals) bands along $\Gamma$-A (z-direction) seems quite small, indicating the weak interlayer coupling among these in-plane orbitals along the stacking direction.

\section{Surface states}

Similar to $\mathrm{Na}_{3} \mathrm{Bi}$, there will be surface states for $\mathrm{DSMs} \mathrm{Na}_{2} \mathrm{MgPb}$ and $\mathrm{Na}_{2} \mathrm{CdSn}$. To simulate surface states to be observed by the angle-resolved photoemission spectroscopy (ARPES), we use an iterative surface Green's function method, ${ }^{31,32}$ where the HSE + SOC band structures are used in generating the maximally localized Wannier functions. The Brillouin zone of bulk and the projected surface Brillouin zones of (100), (010), and (001) planes are exactly the same as those of $\mathrm{Na}_{3} \mathrm{Bi}^{2}{ }^{2}$ WC-type $\mathrm{ZrTe}^{33}$ and $\mathrm{KHgAs}^{34}$ The projected surface density of states for the (100), 
(a) Na2MgSn

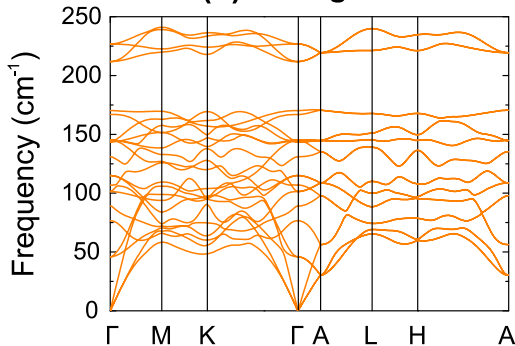

(b) $\mathrm{Na} 2 \mathrm{MgPb}$

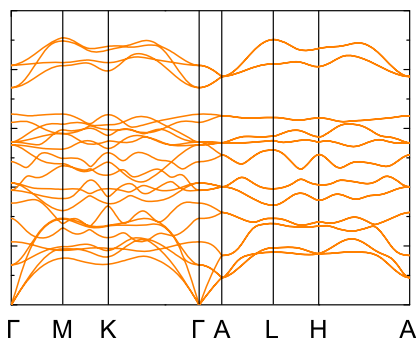

(c) $\mathrm{Na} 2 \mathrm{CdSn}$

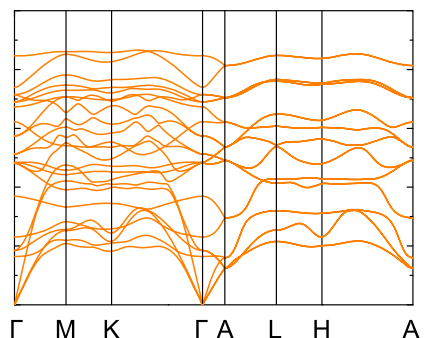

Fig. 2 Phonon dispersion for a $\mathrm{Na}_{2} \mathrm{MgSn}, \mathbf{b} \mathrm{Na}_{2} \mathrm{MgPb}$, and $\mathbf{c} \mathrm{Na}_{2} \mathrm{CdSn}$

(a) Na2MgSn
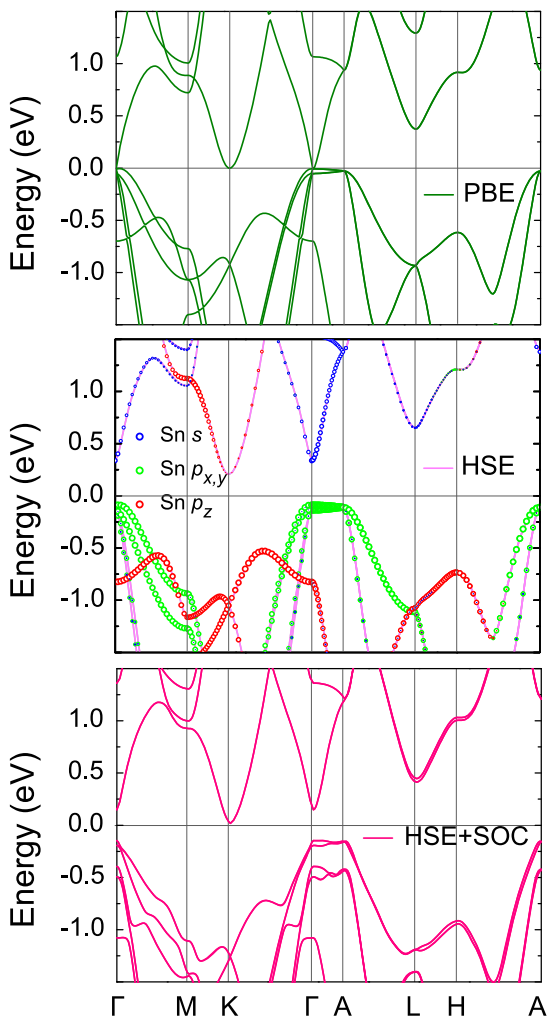

(b) $\mathrm{Na} 2 \mathrm{MgPb}$
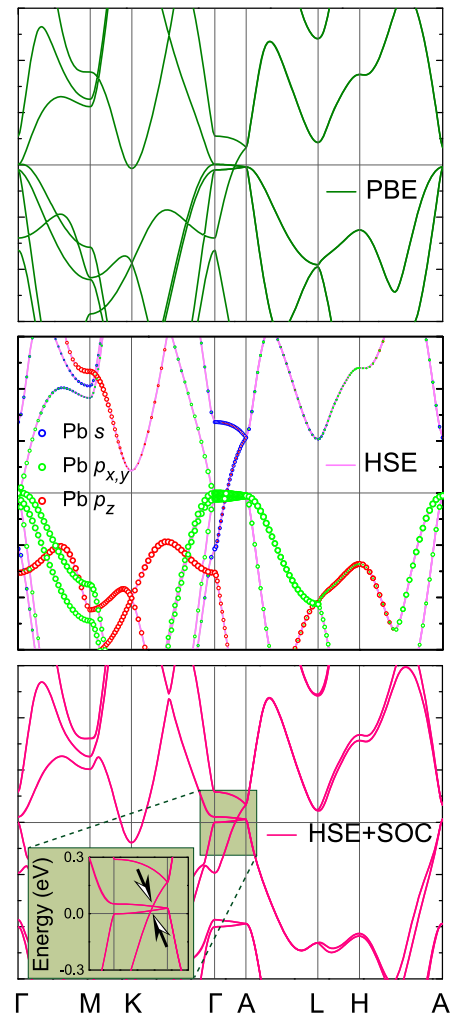

(c) $\mathrm{Na}_{2} \mathrm{CdSn}$
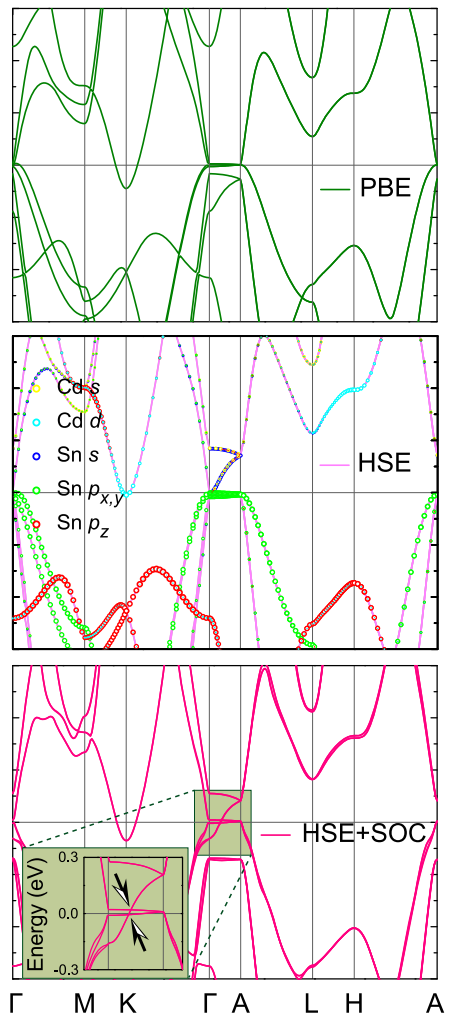

Fig. 3 Calculated electronic structures for a $\mathrm{Na}_{2} \mathrm{MgSn}$, b Na $\mathrm{Na}_{2} \mathrm{MgPb}$, and $\mathbf{c} \mathrm{Na}_{2} \mathrm{CdSn}$ using the PBE functional without spin-orbit coupling (top panel), and hybrid-functional without (middle panel) and with (bottom panel) spin-orbit coupling. The fatted bands with the weight of atomic orbital projection near the Fermi level are present in the middle panel. The two arrows point out the two Dirac cones formed by band crossings from $s$-band and bonding, anti-bonding $p_{x, y}-$ bands along $\Gamma-A$

(010), and (001) surfaces of $\mathrm{Na}_{2} \mathrm{MgPb}$ are shown in Fig. 4(a)-(c). On both (100) and (010) side surfaces, the projection of bulk Dirac cone (pointed by the arrow) is well separated from the topological surface Dirac cone (labeled by the circle). The surface Dirac cone has its branches merging into the bulk states at the projection of 3D Dirac point, which leads to the arc like Fermi surface when the Fermi level is set at the bulk Dirac nodal point. There are two Fermi arcs touch each other at the surface projection of bulk Dirac point at $61 \mathrm{meV}$, as shown in Fig. 4(d, e). For the (001) surface, the projection of bulk Dirac nodes overlaps with the surface Dirac cone as shown in Fig. $4(\mathrm{c})$, which is similar to the case in $\mathrm{Na}_{3} \mathrm{Bi}^{2,4}$

The projected surface density of states for the (100), (010), and (001) surfaces of $\mathrm{Na}_{2} \mathrm{CdSn}$ are shown in Fig. 5. For both the (100) and (010) surfaces, the bulk Dirac cone is closer to the $\Gamma$ point. Due to the smaller band splitting between the bonding and antibonding $p_{x, y}$-orbital bands, the nontrivial surface states of
$\mathrm{Na}_{2} \mathrm{CdSn}$ are not as clear as those in $\mathrm{Na}_{2} \mathrm{MgPb}$. For the (100) surface, the Fermi arcs are hidden within the projection of the bulk states on the surface. They can be well revealed in the Fermi surface plot on the (010) surface with Fermi level at bulk Dirac point of $40 \mathrm{meV}$, as shown in Fig. 5(e). For the (001) surface, the surface projection of the bulk states is superposed with the nontrivial surface states, which is similar to the case in $\mathrm{Na}_{2} \mathrm{MgPb}$.

In this paper, we demonstrate an approach for searching new DSM materials by tuning the chemical degree of freedom based on material design of well-known DSM Na${ }_{3} \mathrm{Bi}$. By keeping both the crystal and electronic structures essentially identical to $\mathrm{Na}_{3} \mathrm{Bi}$, three compounds $\mathrm{Na}_{2} \mathrm{MgSn}, \mathrm{Na}_{2} \mathrm{MgPb}$, and $\mathrm{Na}_{2} \mathrm{CdSn}$ are naturally located and two of them are identified as DSM candidates based on our theoretical calculations. The phonon calculations confirm that these compounds are stable than $\mathrm{Na}_{3} \mathrm{Bi}$, paving the way for experimental verification. The hybrid-functional calculations with 


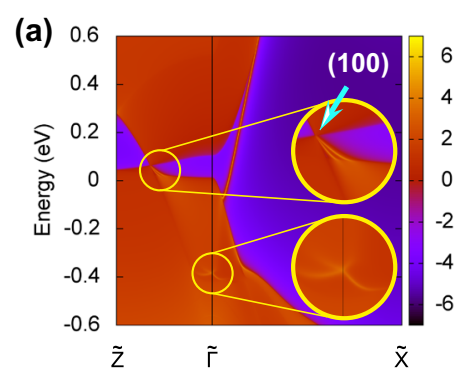

(d) 0

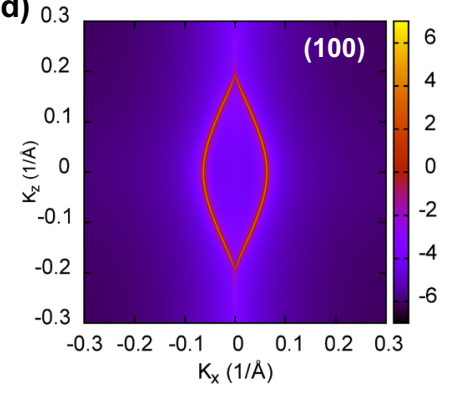

(b) 0.

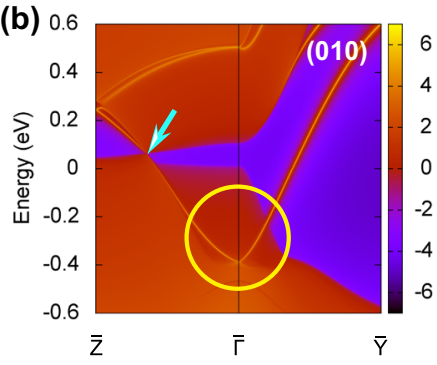

(e) 0.

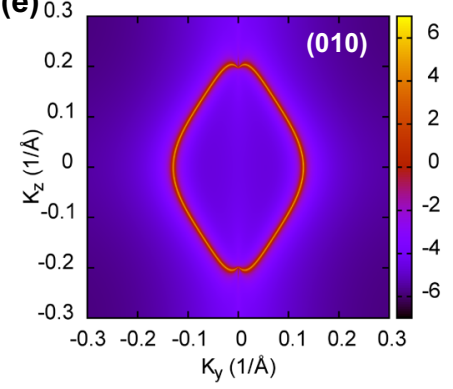

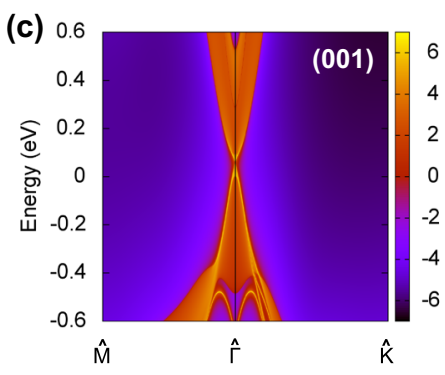

(f) 0.3

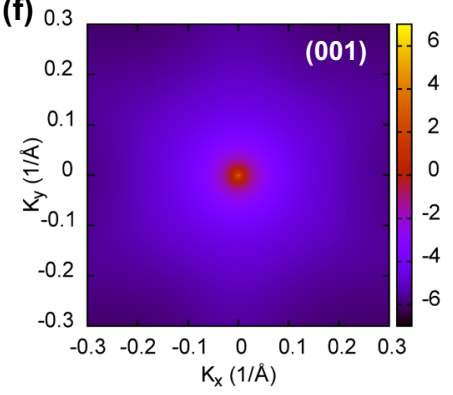

Fig. 4 Surface band structure for a (100), $\mathbf{b}(010)$, and $\mathbf{c}(001)$ surfaces of $\mathrm{Na}_{2} \mathrm{MgPb}$. The arrow points out the bulk Dirac cone, and the circle labels the topological surface states due to $Z_{2}=1$ in $k_{z}=0$ plane. The corresponding Fermi surface with Fermi level at bulk Dirac point $(61 \mathrm{meV})$ is shown in $\mathbf{d}-\mathbf{f}$

(a)

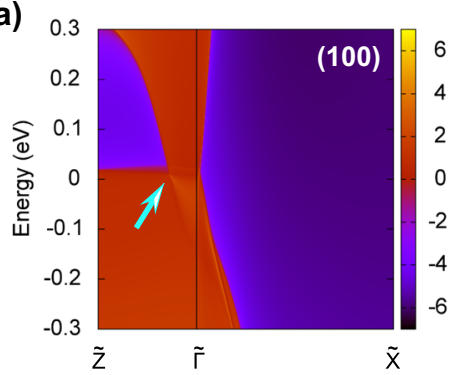

(d)

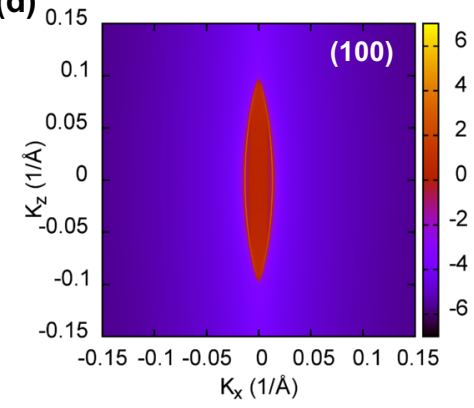

(b)

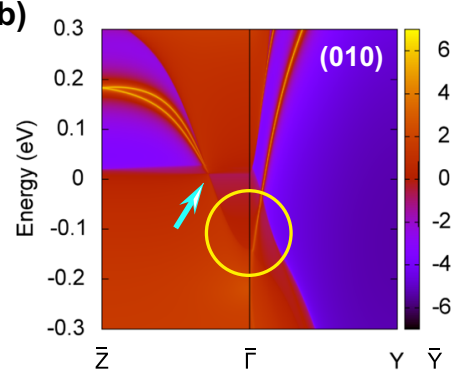

(e) 0

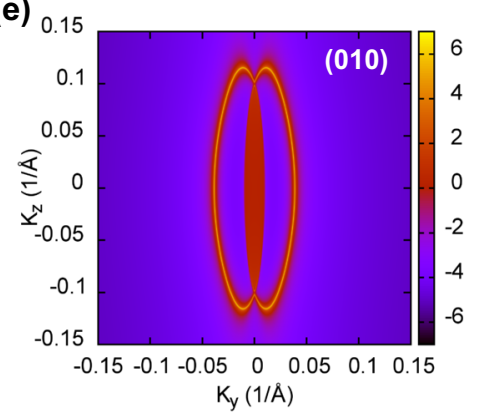

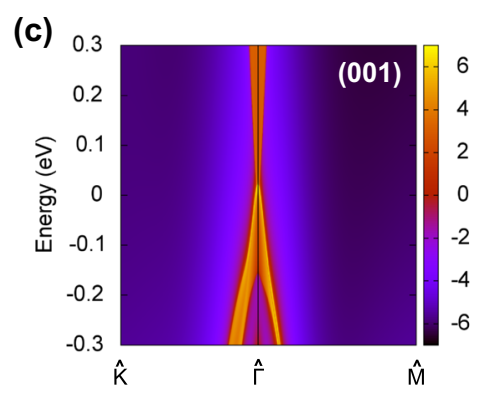

(f)

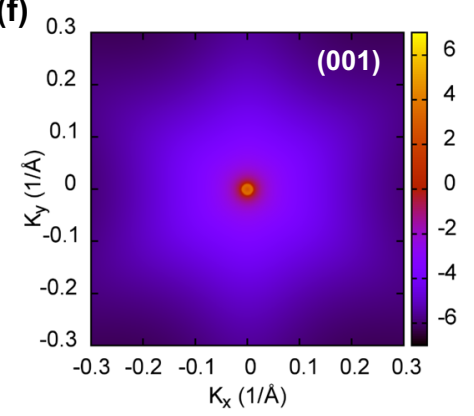

Fig. 5 Surface band structure for a (100), b (010), and c (001) surfaces of $\mathrm{Na}_{2} \mathrm{CdSn}$. The arrow points out the bulk Dirac cone, and the circle labels the topological surface states. The corresponding Fermi surface with Fermi level at bulk Dirac point (40 meV) is shown in $\mathbf{d}-\mathbf{f}$

spin-orbit coupling show that $\mathrm{Na}_{2} \mathrm{MgSn}$ is an indirect band gap normal semiconductor. By substituting $\mathrm{Sn}$ by heavier $\mathrm{Pb}$, the band inversion occurs, and the Dirac nodes due to band crossing are protected by crystal symmetry in $\mathrm{Na}_{2} \mathrm{MgPb}$. For $\mathrm{Na}_{2} \mathrm{CdSn}$, the band inversion is induced by replacing $\mathrm{Mg}$ with heavier $\mathrm{Cd}$ in $\mathrm{Na}_{2} \mathrm{MgSn}$. Moreover, the coexistence of both a bulk 3D Dirac cone and topological surface states can be observed in the projected surface density of states for side surfaces (100) and (010), which can be used as a reference for further experimental validation in ARPES or scanned tunneling microscopy measurements. We hope the idea in this example would lead to more material design efforts based on known topological materials for more successful and efficient predictions.

During the preparation of this manuscript, ref. ${ }^{35}$ proposed that $\mathrm{Na}_{2} \mathrm{CdSn}$ is a topological crystalline insulator (TCl) candidate, which is consistent with our PBE + SOC calculation. From Fig. 3(c), it is seen that both bonding and anti-bonding $s$ bands are lower than the $p_{x, y}$ bands along the whole path $\Gamma-A$. And we have confirmed that in this case it is a TCl of $Z_{12}=8^{36}$ with mirror Chern number 2 in $m_{001}$ plane. 


\section{METHODS}

First-principles calculations are performed using the Vienna ab-initio simulation package (VASP) ${ }^{37}$ based on density functional theory (DFT). The generalized gradient approximation (GGA) in the PBE parameterization for the exchange-correlation functional is used for structural relaxation. A plane-wave basis set is employed with kinetic energy cutoff of $500 \mathrm{eV}$. We use the projector-augmented-wave method and the related pseudopotential for each element. A $11 \times 11 \times 5$ q-mesh is used during structural relaxation for the unit-cell until the energy difference is converged within $10^{-6} \mathrm{eV}$, with a Hellman-Feynman force convergence threshold of $10^{-4} \mathrm{eV} /$ $\AA$. To improve the underestimation of band gap in the PBE functional, hybrid-functional method based on the HSE method are adopted. ${ }^{38-40}$ The harmonic interatomic force constants (IFCs) are obtained by density functional perturbation theory using a $3 \times 3 \times 2$ supercell with a $3 \times 3 \times 3$ qmesh. The phonon dispersion is calculated from the harmonic IFCs using the PHONOPY code. ${ }^{41,42}$ The Wannier functions ${ }^{43}$ for $\mathrm{Cd} / \mathrm{Mg} s$-orbital and $\mathrm{Sn} / \mathrm{Pb} s$-and $p$-orbitals are generated, which are used in the surface state calculations.

\section{DATA AVAILABILITY}

The datasets generated during and/or analyzed during the current study are available from the corresponding author on reasonable request.

\section{ACKNOWLEDGEMENTS}

We thank the valuable discussions with X. Wan, C. Fang, Z. Song, and T. Zhang. This work is supported by the the National Key Research and Development Program of China (No. 2016YFA0300600 and 2018YFA0305700), the National Natural Science Foundation of China (Grant Nos. 11374063 and 11674369) and the "Strategic Priority Research Program (B)" of the Chinese Academy of Sciences (Grant No. XDB07020100).

\section{AUTHOR CONTRIBUTIONS}

H.M.W. and H.Z. designed the research. B.P., C.M.Y., and H.Z. performed the calculations. B.P., H.M.W., Z.F., and H.Z. analyzed and discussed the results. B.P. and W. H.M. wrote the text of the manuscript. B.P. and C.M.Y. contributed equally to this work. All authors commented on the manuscript.

\section{ADDITIONAL INFORMATION}

Competing Interests: The authors declare no competing interests.

Publisher's note: Springer Nature remains neutral with regard to jurisdictional claims in published maps and institutional affiliations.

\section{REFERENCES}

1. Young, S. M. et al. Dirac semimetal in three dimensions. Phys. Rev. Lett. 108, 140405 (2012).

2. Wang, Z. et al. Dirac semimetal and topological phase transitions in $A_{3} B i(A=N a$, K, Rb). Phys. Rev. B 85, 195320 (2012).

3. Wang, Z., Weng, H., Wu, Q., Dai, Xi \& Fang, Z. Three-dimensional Dirac semimetal and quantum transport in $\mathrm{Cd}_{3} \mathrm{As}_{2}$. Phys. Rev. B 88, 125427 (2013).

4. Weng, H., Dai, Xi \& Fang, Z. Topological semimetals predicted from first-principles calculations. J. Phys.: Condens. Matter 28, 303001 (2016).

5. Armitage, N. P. et al. Weyl and Dirac semimetals in three-dimensional solids. Rev. Mod. Phys. 90, 015001 (2018).

6. Novoselov, K. S. et al. Electric field effect in atomically thin carbon films. Science 306, 666 (2004).

7. Liu, Z. K. et al. Discovery of a three-dimensional topological dirac semimetal, $\mathrm{Na}_{3} \mathrm{Bi}$. Science 343, 864 (2014).

8. Bradlyn, B. et al. Beyond Dirac and Weyl fermions: Unconventional quasiparticles in conventional crystals. Science 353, aaf5037 (2016).

9. Bernevig, A., Weng, H., Fang, Z. \& Dai, Xi Recent progress in the study of topological semimetals. J. Phys. Soc. Jpn. 87, 041001 (2018).

10. Orlita, M. et al. Observation of three-dimensional massless Kane fermions in a zinc-blende crystal. Nat. Phys. 10, 233 (2014)

11. Yang, B. -J. \& Nagaosa, N. Classification of stable three-dimensional Dirac semimetals with nontrivial topology. Nat. Commun. 5, 4898 (2014).
12. Murakami, S. Phase transition between the quantum spin Hall and insulator phases in 3D: emergence of a topological gapless phase. New J. Phys. 9, 356 (2007).

13. Weng, H. Fang, C., Fang, Z. \& Dai, X. A new member in topological semimetals family. Natl Sci. Rev. 4, 798-799 (2017).

14. Zdanowicz, W. \& Zdanowicz, L. Semiconducting compounds of the All BV group. Annu. Rev. Mater. Sci. 5, 301-328 (1975).

15. Wan, X., Turner, A. M., Vishwanath, A. \& Savrasov, S. Y. Topological semimetal and Fermi-arc surface states in the electronic structure of pyrochlore iridates. Phys. Rev. B 83, 205101 (2011).

16. Xiong, J. et al. Evidence for the chiral anomaly in the Dirac semimetal $\mathrm{Na}_{3} \mathrm{Bi}$. Science 350, 413 (2015).

17. Gorbar, E. V., Miransky, V. A. \& Shovkovy, I. A. Chiral anomaly, dimensional reduction, and magnetoresistivity of Weyl and Dirac semimetals. Phys. Rev. B 89, 085126 (2014).

18. Liu, Z. K. et al. A stable three-dimensional topological Dirac semimetal $\mathrm{Cd}_{3} \mathrm{As}_{2}$. Nat. Mater. 13, 677-681 (2014).

19. Borisenko, S. et al. Experimental realization of a three-dimensional Dirac semimetal. Phys. Rev. Lett. 113, 027603 (2014).

20. Jeon, S. et al. Landau quantization and quasiparticle interference in the threedimensional Dirac-semimetal $\mathrm{Cd}_{3} \mathrm{As}_{2}$. Nat. Mater. 13, 851-856 (2014).

21. Neupane, M. et al. Observation of a three-dimensional topological Dirac semimetal phase in high-mobility $\mathrm{Cd}_{3} \mathrm{As}_{2}$. Nat. Commun. 5, 3786 (2014).

22. Yamada, T., Deringer, V. L., Dronskowski, R. \& Yamane, H. Synthesis, crysta structure, chemical bonding, and physical properties of the ternary $\mathrm{Na} / \mathrm{Mg}$ stannide $\mathrm{Na}_{2} \mathrm{MgSn}$. Inorg. Chem. 51, 4810-4816 (2012).

23. Yamada, T. et al. Synthesis, crystal structure, and high-temperature phase transition of the novel plumbide $\mathrm{Na}_{2} \mathrm{MgPb}$. Inorg. Chem. 53, 5253-5259 (2014).

24. Matthes, H. \& Schuster, R. Ternary sodium phases with cadmium or mercury and tin or lead. Z. für Naturforsch. B 35, 778-780 (1980).

25. Zhang, R. F. et al. Stability and strength of transition-metal tetraborides and triborides. Phys. Rev. Lett. 108, 255502 (2012).

26. Zhou, L. et al. Structural stability and thermodynamics of $\mathrm{CrN}$ magnetic phases from ab initio calculations and experiment. Phys. Rev. B 90, 184102 (2014).

27. Peng, Bo et al. Stability and strength of atomically thin borophene from first principles calculations. Mater. Res. Lett. 5, 399-407 (2017).

28. Cheng, X. et al. Ground-state phase in the three-dimensional topological Dirac semimetal $\mathrm{Na}_{3}$ Bi. Phys. Rev. B 89, 245201 (2014).

29. Cheng, X., Li, R., Li, D., Li, Y. \& Chen, X. -Q. Stable compositions and structures in the Na-Bi system. Phys. Chem. Chem. Phys. 17, 6933-6947 (2015).

30. Shao, D. et al. Strain-induced quantum topological phase transitions in $\mathrm{na}_{3} \mathrm{Bi}$ Phys. Rev. B 96, 075112 (2017).

31. Zhang, W., Yu, R., Zhang, H. -J., Dai, Xi \& Fang, Z. First-principles studies of the three-dimensional strong topological insulators $\mathrm{Bi}_{2} \mathrm{Te}_{3}, \mathrm{Bi}_{2} \mathrm{Se}_{3}$ and $\mathrm{Sb}_{2} \mathrm{Te}_{3}$. New J. Phys. 12, 065013 (2010).

32. Wu, QuanSheng, Zhang, ShengNan, Song, H. -F., Troyer, M. \& Soluyanov, A. A WannierTools: An open-source software package for novel topological materials. Comput. Phys. Commun. 224, 405-416 (2018).

33. Weng, H., Fang, C., Fang, Z. \& Dai, Xi Coexistence of weyl fermion and massless triply degenerate nodal points. Phys. Rev. B 94, 165201 (2016).

34. Wang, Z., Alexandradinata, A., Cava, R. J. \& Bernevig, B. A. Hourglass fermions. Nature 532, 189- (2016).

35. Tang, F., Po, H. C., Vishwanath, A. \& Wan, X. Topological materials discovery by largeorder symmetry indicators, arXiv 1806, 04128 (2018).

36. Song, Z., Zhang, T., Fang, Z. \& Fang, C. Quantitative mappings between symmetry and topology in solids. Nat. Commun. 9, 3530 (2018).

37. Kresse, G. \& Furthmüller, J. Efficient iterative schemes for ab initio total-energy calculations using a plane-wave basis set. Phys. Rev. B 54, 11169-11186 (1996).

38. Heyd, J., Scuseria, G. E. \& Ernzerhof, M. Hybrid functionals based on a screened Coulomb potential. J. Chem. Phys. 118, 8207 (2003).

39. Heyd, J., Scuseria, G. E. \& Ernzerhof, M. Erratum: ybrid functionals based on a screened Coulomb potential?. J. Chem. Phys. 124, 219906 (2006).

40. Peralta, J. E., Heyd, J., Scuseria, G. E. \& Martin, R. L. Spin-orbit splittings and energy band gaps calculated with the Heyd-Scuseria-Ernzerhof screened hybrid functional. Phys. Rev. B 74, 073101 (2006).

41. Togo, A., Oba, F. \& Tanaka, I. First-principles calculations of the ferroelastic transition between rutile-type and $\mathrm{CaCl}_{2}$-type $\mathrm{SiO}_{2}$ at high pressures. Phys. Rev. $B$ 78, 134106 (2008).

42. Togo, A. \& Tanaka, I. First principles phonon calculations in materials science. Scr. Mater. 108, 1-5 (2015).

43. Mostofi, A. A. et al. An updated version of Wannier90: A tool for obtaining maximallylocalised Wannier functions. Comput. Phys. Commun. 185, 2309-2310 (2014). 
Open Access This article is licensed under a Creative Commons Attribution 4.0 International License, which permits use, sharing, adaptation, distribution and reproduction in any medium or format, as long as you give appropriate credit to the original author(s) and the source, provide a link to the Creative Commons license, and indicate if changes were made. The images or other third party material in this article are included in the article's Creative Commons license, unless indicated otherwise in a credit line to the material. If material is not included in the article's Creative Commons license and your intended use is not permitted by statutory regulation or exceeds the permitted use, you will need to obtain permission directly from the copyright holder. To view a copy of this license, visit http://creativecommons. org/licenses/by/4.0/.

(c) The Author(s) 2018 